\title{
Bloch Equations Revisited: New Analytical Solutions for the Generalized Bloch Equations
}

\section{P. K. MADHU ${ }^{1}$ and ANIL KUMAR ${ }^{1,2, *}$}

${ }^{1}$ Department of Physics and ${ }^{2}$ Sophisticated Instruments Facility, Indian Institute of Science, Bangalore- 560012 , India

\begin{abstract}
The generalized Bloch equations in the rotating frame are solved in Cartesian space by an approach that is different from the earlier Torrey solutions. The solutions are cast into a compact and convenient matrix notation, which paves the way for a direct physical insight and comprehension of the evolution of various magnetization components. The solutions are expressed as a sum of two terms: One describes the decay of the initial state; the other describes the growth of the steady state. The representative trajectories of each component of the above terms plotted separately describe the complete time evolution of each magnetization component. @ 1997 John Wiley \& Sons, Inc.
\end{abstract}

\section{INTRODUCTION}

In 1946, soon after the experimental discovery of magnetic resonance, Felix Bloch gave phenomenological equations of motion for various magnetization components (1). These have played a central role in elucidating magnetic resonance ever since. The generalized form of the Bloch equations contains an offresonance radio frequency (rf) field, which couples all the equations. An analytical solution of the coupled equations was given by Torrey in 1949 (2), which to date is the last word on their solution. Despite the availability of this general solution, $a b$ initio solutions of the coupled equations have been given in the lit-

* To whom correspondence should be addressed. Tel: 91-803092724; (R) 91-80-3342401, Fax: 91-80-3341683; 3342085, Email: anilnmr@physics.iisc.ernet.in; madhu@physics.iisc. ernet.in. erature for various limiting situations, such as steadystate solutions $(3,4)$, on-resonance solutions $(5,6)$, solutions neglecting relaxation (7), and those that involve weak rf fields (8). The reasons for these later solutions are that they are easier to interpret and give more physical insight than do the Torrey solutions. Recently, several publications have restated and completed Torrey's solutions - and remedied typographical errors $(9,10)$. Alternatively, the generalized coupled equations are solved numerically (11).

We have given analytical solution to the generalized Bloch equations in the rotating frame without any approximations (12). We also redefine various constants, which facilitates setting independent limits on various parameters. Another feature of our solution is that it provides clear insight about the development of various magnetization components and their interconversions. This insight is made possible by casting the 
Table 1 Matrix Elements of $\mathbf{A}$

$$
\begin{aligned}
& \boldsymbol{A}_{11}=\left[U_{1} e^{\lambda_{1} t}+e^{\mu_{2} t}\left(\left(1-U_{1}\right) \cos \mu_{3} t+U_{2} \sin \mu_{3} t\right)\right] \\
& \boldsymbol{A}_{12}=-\boldsymbol{A}_{21}=\left[\boldsymbol{N}_{1} e^{\lambda_{1} t}-e^{\mu_{2} t}\left(N_{1} \cos \mu_{3} t-N_{2} \sin \mu_{3} t\right)\right] \\
& \boldsymbol{A}_{13}=\boldsymbol{A}_{31}=\left[\boldsymbol{N}_{3} e^{\lambda_{1} t}-e^{\mu_{2} t}\left(\boldsymbol{N}_{3} \cos \mu_{3} t-\boldsymbol{N}_{4} \sin \mu_{3} t\right)\right] \\
& \boldsymbol{A}_{22}=\left[V_{1} e^{\lambda_{1} t}+e^{\mu_{2} t}\left(\left(1-V_{1}\right) \cos \mu_{3} t+V_{2} \sin \mu_{3} t\right)\right] \\
& \boldsymbol{A}_{23}=-\boldsymbol{A}_{32}=\left[\boldsymbol{N}_{5} e^{\lambda_{1} t}-e^{\mu_{2} t}\left(N_{5} \cos \mu_{3} t-N_{6} \sin \mu_{3} t\right)\right] \\
& \boldsymbol{A}_{33}=\left[Z_{1} e^{\lambda_{1} t}+e^{\mu_{2} t}\left(\left(1-Z_{1}\right) \cos \mu_{3} t+Z_{2} \sin \mu_{3} t\right)\right]
\end{aligned}
$$

solution into a compact matrix notation as a sum of two terms-one describes the interconversion of the various magnetization components of the initial state and their decay to zero, and the other describes the independent growth of the steady state. Such a separation is extremely useful, particularly because it provides physical insight about the development of the various magnetization components. It may also be pointed out that these two terms can be separated by the use of two-dimensional (2D) experiments.

From our solution, we have earlier extracted the time development of each magnetization component, which is a sum of the contribution from the initial state, interconversion of various components, and the growth of the steady state (12). It turns out that it is also quite illustrative to monitor separately the time development of each of the above elements of each magnetization component-to monitor the time development of the various elements of the above matrices. In this article, we discuss the time development of various elements of the above matrices to illustrate in a pedagogical manner the new solution of the Bloch equations.

\section{BLOCH EQUATIONS AND THEIR SOLUTION}

The phenomenological Bloch equations in vector notation in the laboratory frame are given by [1]

$$
\frac{d \mathbf{M}}{d t}=\gamma \mathbf{M} \times \mathbf{H}-\frac{\boldsymbol{M}_{x}}{T_{2}} \vec{i}-\frac{\boldsymbol{M}_{y}}{T_{2}} \vec{j}-\frac{\boldsymbol{M}_{z}-\boldsymbol{M}_{0}}{T_{1}} \vec{k}
$$

where $\mathbf{M}$ is the total magnetization vector having components $\boldsymbol{M}_{x}, \boldsymbol{M}_{y}$ and $\boldsymbol{M}_{z} ; \boldsymbol{M}_{0}$ is the equilibrium $z$ magnetization in the absence of an rf field; $\gamma$ is the magnetogyric ratio; and $T_{1}$ and $T_{2}$ are, respectively, the spin-lattice and spin-spin relaxation time con-

\section{Table 2 Elements of Vector B}

$$
\begin{aligned}
& \boldsymbol{B}_{11}=\left[1-\boldsymbol{M}_{1} e^{\lambda_{1} t}-e^{\mu_{2} t}\left(\left(1-\boldsymbol{M}_{1}\right) \cos \mu_{3} t-\boldsymbol{M}_{2} \sin \mu_{3} t\right)\right] \\
& \boldsymbol{B}_{22}=\left[1-\boldsymbol{M}_{3} e^{\lambda_{1} t}-e^{\mu_{2} t}\left(\left(1-\boldsymbol{M}_{3}\right) \cos \mu_{3} t-\boldsymbol{M}_{4} \sin \mu_{3} t\right)\right] \\
& \boldsymbol{B}_{33}=\left[1-\boldsymbol{M}_{5} e^{\lambda_{1} t}-e^{\mu_{2} t}\left(\left(1-\boldsymbol{M}_{5}\right) \cos \mu_{3} t-\boldsymbol{M}_{6} \sin \mu_{3} t\right)\right]
\end{aligned}
$$

Table 3 Expressions for U, V, Z, N, and M Characterizing the Elements of $A$ and $B$

$$
\begin{aligned}
U_{1}= & \mu_{3} L\left[\mu_{2}^{2}+\mu_{3}^{2}+\beta^{2}-\delta^{2}+2 \beta \mu_{2}\right] \\
U_{2}= & -L\left[\lambda_{1}^{2}\left(\beta+\mu_{2}\right)+\left(\beta+\lambda_{1}\right)\left(\mu_{3}^{2}-\mu_{2}^{2}\right)\right. \\
& \left.+\left(\beta^{2}-\delta^{2}\right)\left(\lambda_{1}-\mu_{2}\right)\right] \\
V_{1}= & \mu_{3} L\left[\mu_{2}^{2}+\mu_{3}^{2}+\beta^{2}-\delta^{2}-\omega_{1}^{2}+2 \mu_{2} \beta\right] \\
V_{2}= & -L\left[\lambda_{1}^{2}\left(\beta+\mu_{2}\right)+\left(\beta+\lambda_{1}\right)\left(\mu_{3}^{2}-\mu_{2}^{2}\right)\right. \\
& \left.+\left(\beta^{2}-\delta^{2}-\omega_{1}^{2}\right)\left(\lambda_{1}-\mu_{2}\right)\right] \\
Z_{1}= & \mu_{3} L\left[\mu_{2}^{2}+\mu_{3}^{2}+\alpha^{2}-\omega_{1}^{2}+2 \mu_{2} \alpha\right] \\
Z_{2}= & -L\left[\lambda_{1}^{2}\left(\alpha+\mu_{2}\right)+\left(\alpha+\lambda_{1}\right)\left(\mu_{3}^{2}-\mu_{2}^{2}\right)\right. \\
& \left.+\left(\alpha^{2}-\omega_{1}^{2}\right)\left(\lambda_{1}-\mu_{2}\right)\right] \\
N_{1}= & 2 \delta \mu_{3} L\left(\beta+\mu_{2}\right) \\
N_{2}= & -\delta L\left[2 \beta\left(\lambda_{1}-\mu_{2}\right)+\left(\lambda_{1}^{2}+\mu_{3}^{2}-\mu_{2}^{2}\right)\right] \\
N_{3}= & \delta \omega_{1} \mu_{3} L \\
N_{4}= & -\delta \omega_{1} L\left(\lambda_{1}-\mu_{2}\right) \\
N_{5}= & \omega_{1} \mu_{3} L\left(\alpha+\beta+2 \mu_{2}\right) \\
N_{6}= & -\omega_{1} L\left[(\alpha+\beta)\left(\lambda_{1}-\mu_{2}\right)-\left(\mu_{2}^{2}-\mu_{3}^{2}-\lambda_{1}^{2}\right)\right] \\
M_{1}= & \mu_{3} L\left(\mu_{2}^{2}+\mu_{3}^{2}\right) \\
M_{2}= & \lambda_{1} L\left(\lambda_{1} \mu_{2}+\mu_{3}^{2}-\mu_{2}^{2}\right) \\
M_{3}= & \mu_{3} L\left[\left(\mu_{2}^{2}+\mu_{3}^{2}\right)\left(\beta+\lambda_{1}\right)\right] / \beta \\
M_{4}= & \lambda_{1} L\left[\beta\left(\lambda_{1} \mu_{2}+\mu_{3}^{2}-\mu_{2}^{2}\right)+\left(\lambda_{1}-\mu_{2}\right)\left(\mu_{2}^{2}+\mu_{3}^{2}\right)\right] / \beta \\
M_{5}= & -\mu_{3}\left(\mu_{2}^{2}+\mu_{3}^{2}\right) L\left[\lambda_{1}\left(\alpha+2 \mu_{2}\right)-\left(\beta^{2}+\delta^{2}\right)\right] /\left(\beta^{2}+\delta^{2}\right) \\
M_{6}= & \lambda_{1} L\left\{\left(\beta^{2}+\delta^{2}\right)\left(\lambda_{1} \mu_{2}+\mu_{3}^{2}-\mu_{2}^{2}\right)-\left[\left(\lambda_{1}-\mu_{2}\right) \alpha\right.\right. \\
& \left.\left.-\left(\mu_{2}^{2}-\mu_{3}^{2}-\lambda_{1}^{2}\right)\right]\left(\mu_{2}^{2}+\mu_{3}^{2}\right)\right\} /\left(\beta^{2}+\delta^{2}\right) \\
\text { where } & L=1 / \mu_{3}\left[\left(\mu_{2}-\lambda_{1}\right)^{2}+\mu_{3}^{2}\right] \\
& \lambda_{1}, \lambda_{2}=\mu_{1}+i \lambda_{2}
\end{aligned}
$$

Here $\lambda_{1}, \lambda_{2}=\mu_{2}+i \mu_{3}$, and $\lambda_{3}=\mu_{2}-i \mu_{3}$ are the roots of the cubic Eq. [3-1] (obtained from text Eqs. [3]-[5]).

$$
\lambda^{3}+a \lambda^{2}+b \lambda+c=0
$$

where

$$
\begin{aligned}
& \mathrm{a}=\alpha+2 \beta \\
& \mathrm{b}=\beta^{2}+\delta^{2}+\omega_{1}^{2}+2 \alpha \beta \\
& \mathrm{c}=\alpha \beta^{2}+\alpha \delta^{2}+\beta \omega_{1}^{2}
\end{aligned}
$$

with $\lambda_{1}$ and $\mu_{2}$ real and negative. The roots of Eq. [3-1] are contained in standard textbooks $(13,14)$ and reproduced in Refs. 9, 10, and 12 .

stants. $\mathbf{H}$ describes the applied magnetic field, which, in the presence of a steady field along the $z$ axis and an $\mathrm{rf}$ field along the $x$ axis, is given by

$$
\begin{aligned}
& \boldsymbol{H}_{x}=\boldsymbol{H}_{1} \cos \omega t \\
& \boldsymbol{H}_{y}=-\boldsymbol{H}_{1} \sin \omega t \\
& \boldsymbol{H}_{z}=\boldsymbol{H}_{0}
\end{aligned}
$$

\section{Table 4 Steady-State Values of Each} Magnetization Component

$$
\begin{gathered}
u^{\infty}=\frac{\gamma \boldsymbol{H}_{1} \delta T_{2}^{2} \boldsymbol{M}_{\mathrm{o}}}{1+\delta^{2} T_{2}^{2}+\left(\gamma \boldsymbol{H}_{1}\right)^{2} T_{1} T_{2}} \\
\boldsymbol{v}^{\infty}=-\frac{\gamma \boldsymbol{H}_{1} T_{2} \boldsymbol{M}_{\mathrm{o}}}{1+\delta^{2} T_{2}^{2}+\left(\gamma \boldsymbol{H}_{1}\right)^{2} T_{1} T_{2}} \\
\boldsymbol{M}_{z}^{\infty}=\frac{\left(1+\delta^{2} T_{2}^{2}\right) \boldsymbol{M}_{\mathrm{o}}}{1+\delta^{2} T_{2}^{2}+\left(\gamma \boldsymbol{H}_{1}\right)^{2} T_{1} T_{2}}
\end{gathered}
$$




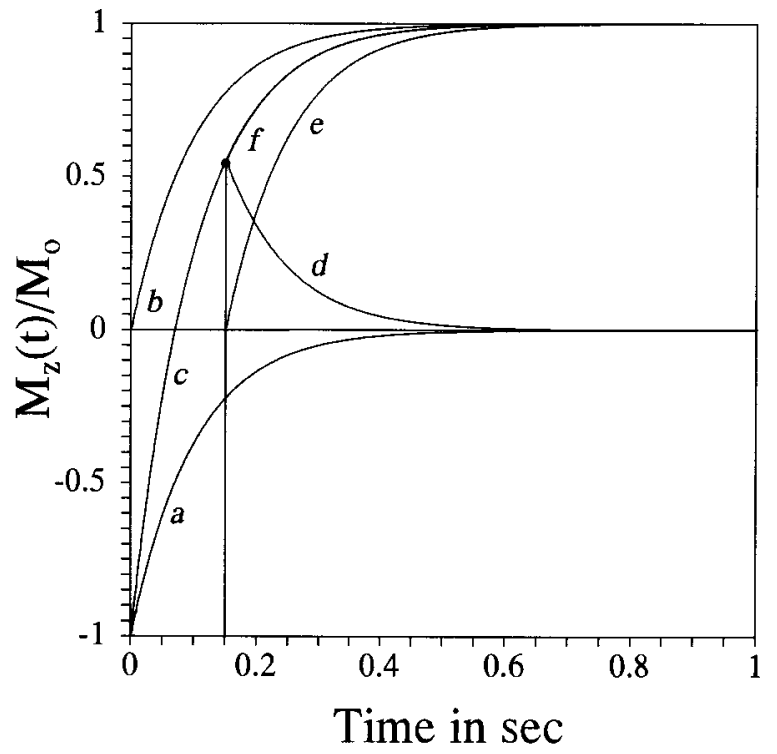

Figure 1 Recovery of inverted magnetization in the absence of an $\mathrm{rf}$ field from Eq. [9] for $T_{1}=0.1 \mathrm{~s}$. Curve (a) shows the decay of the inverted initial state to zero, curve (b) shows the growth of the steady state from zero to unity, curve $(c)$ shows the sum of curves $(a)$ and $(b)$ showing the recovery of the total magnetization. Equation [9] is valid at all points of time. For example, assuming this experiment is interrupted (in a thought experiment) at $t=0.15 \mathrm{~s}$, the total magnetization at that point (indicated by 0 ) then becomes the initial state, which, according to Eq. [9], decays to zero, given by curve $(d)$. Again, the steady state grows from zero, indicated by curve $(e)$; the total is given by curve $(f)$, which is identical to curve $(c)$.

These equations, when transformed into a rotating frame at an angular velocity $\omega$ about the $z$ axis, become

$$
\begin{aligned}
\frac{d u}{d t}+\beta u+\delta v & =0 \\
\frac{d v}{d t}+\beta v-\delta u+\omega_{1} \boldsymbol{M}_{z} & =0 \\
\frac{d \boldsymbol{M}_{z}}{d t}+\alpha \boldsymbol{M}_{z}-\omega_{1} v & =\alpha \boldsymbol{M}_{0}
\end{aligned}
$$

where the $x$ and $y$ components of $\mathbf{M}$ in the laboratory frame are related to $u$ and $v$ in the rotating frame, for positive $\gamma$, by $(1)$

$$
\begin{aligned}
& \boldsymbol{M}_{x}=u \cos \omega t-v \sin \omega t \\
& \boldsymbol{M}_{y}=-(v \cos \omega t+u \sin \omega t)
\end{aligned}
$$

Equations [3]-[5] have been written by using the parameters

$$
\begin{aligned}
& \alpha=\frac{1}{T_{1}} \\
& \beta=\frac{1}{T_{2}} \\
& \delta=\gamma \boldsymbol{H}_{0}-\omega \\
& \omega_{1}=\gamma \boldsymbol{H}_{1}
\end{aligned}
$$

Note that the definitions, Eq. [8] are different from those of Bloch (1), Torrey (2), and Morris and Chilvers (10). They define the constants in dimensionless units by dividing each of the above by $\gamma \boldsymbol{H}_{1}$. The above definitions facilitate independent variation of
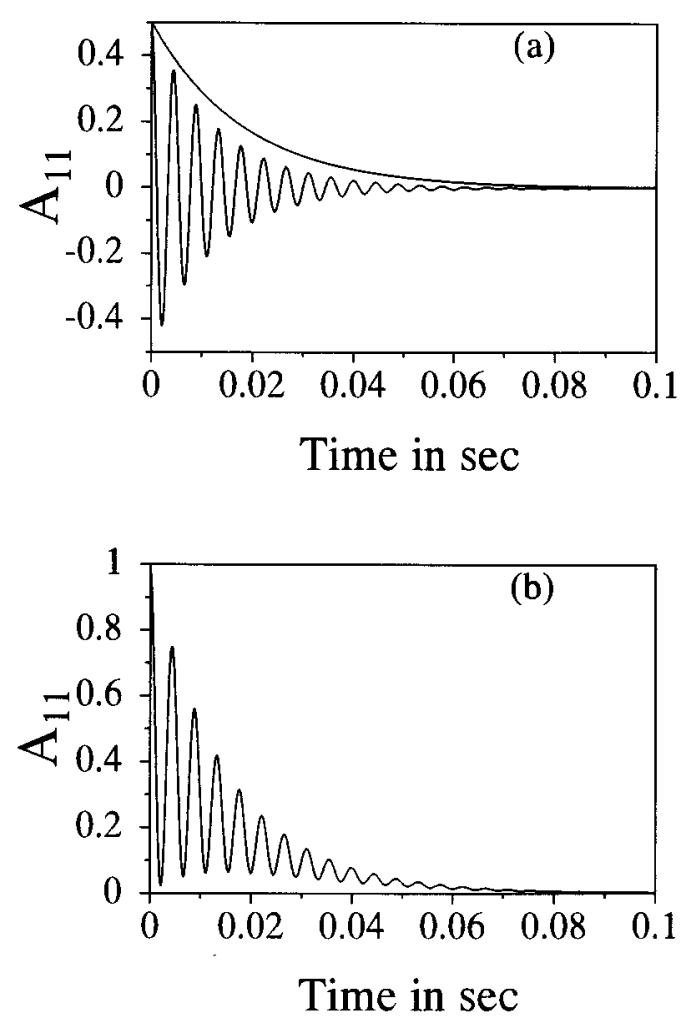

Figure 2 Oscillatory and nonoscillatory parts of $\boldsymbol{A}_{11}$ (Table 1). (a) Separately and (b) added together, for $\omega_{1}=$ $1 \mathrm{KHz}, \delta=1 \mathrm{KHz}, T_{1}=0.1 \mathrm{~s}$, and $T_{2}=0.01 \mathrm{~s}$. For these values, the parameters $U_{1}$ and $U_{2}$ are obtained as $U_{1}=0.50$ and $U_{2}=0.024 . A_{11}$ has large nonoscillatory and oscillatory cosine parts and a small oscillatory sine part. 


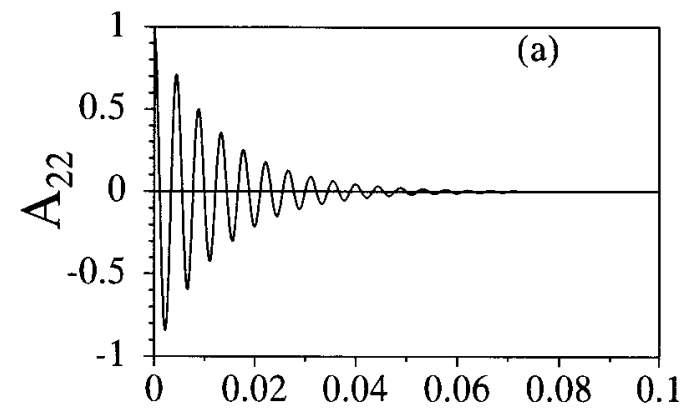

Time in sec

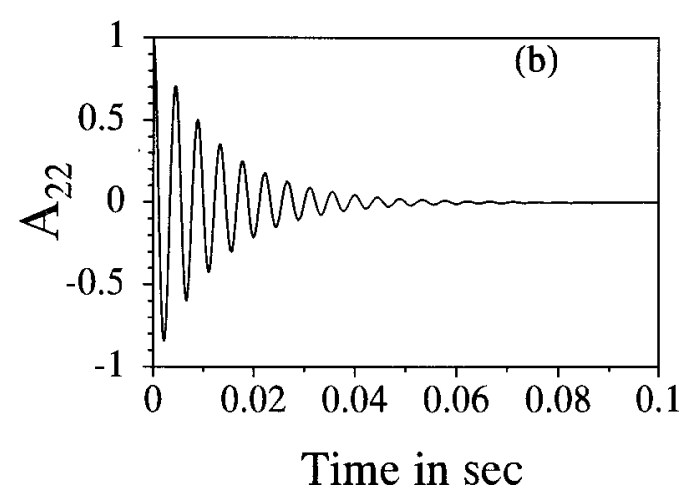

Figure 3 Oscillatory and nonoscillatory parts of $\boldsymbol{A}_{22}$ (Table 1). (a) Separately and (b) added together, for the same values of constants as in Fig. 2. For these values, the parameters $V_{1}$ and $V_{2}$ are obtained as $V_{1}=-0.0011$ and $V_{2}$ $=-0.0159 . \boldsymbol{A}_{22}$ contains mainly the cosine part.

each parameter, including the independent setting of $\gamma \boldsymbol{H}_{1}, 1 / T_{1}$, or $1 / T_{2} \rightarrow 0$.

These coupled, inhomogeneous differential equations (Eqs. [3]-[5]) have been solved by a direct method, expressed in compact matrix notation as follows (12):

$$
\mathbf{M}(t)=\mathbf{A} \mathbf{M}_{0}+\mathbf{B} \mathbf{M}^{\infty}
$$

where

$$
\begin{aligned}
\mathbf{M}(t) & =\left(\begin{array}{c}
u(t) \\
v(t) \\
M_{z}(t)
\end{array}\right) \\
\boldsymbol{M}_{0} & =\left(\begin{array}{c}
u_{0} \\
v_{0} \\
m_{0}
\end{array}\right) \\
\mathbf{M}^{\infty} & =\left(\begin{array}{c}
u^{\infty} \\
v^{\infty} \\
M_{z}^{\infty}
\end{array}\right)
\end{aligned}
$$

The vector $\boldsymbol{M}_{0}$ describes a general initial state and $\mathbf{M}^{\infty}$ the steady state. $\mathbf{A}$ and $\mathbf{B}$ are $3 \times 3$ matrices, with $\mathbf{B}$ turning out to be a diagonal matrix. The various elements of $\mathbf{A}$ and $\mathbf{B}$ are listed in Tables 1 and 2; the parameters of Tables 1 and 2 are given in Table 3 . The values of the various components of $\boldsymbol{M}^{\infty}$ are given in Table 4, and they match the well-known steady-state solutions of the Bloch equation $(3,4)$. (The brackets in the numerator of the expression for $\boldsymbol{M}_{z}^{\infty}$ in Eq. [17] of Ref. 12 are missing.) The general characteristics of the $\mathbf{A}$ and $\mathbf{B}$ matrices are that the $\mathbf{A}$ matrix decays from unity to zero, and the $\mathbf{B}$ matrix grows from zero to unity. The solutions, however, depend on the eigenvalues $\lambda_{1}, \lambda_{2}$, and $\lambda_{3}$ of the cubic equation (Table 3 ; Eq. [3-1]), which in general yields one real negative root $\lambda_{1}$, and two complex, conjugate roots, $\lambda_{2}$ and $\lambda_{3}$. Expressing $\mu_{2}=\left(\lambda_{2}+\lambda_{3}\right) / 2$ and $\mu_{3}=\left(\lambda_{2}-\lambda_{3}\right) / 2 i$, one sees from Tables 1-3 that, while $\lambda_{1}$ and $\mu_{2}$ govern, respectively, the time evolution of the nonoscillatory and the oscillatory parts of $\mathbf{A}$ and $\mathbf{B}, \mu_{3}$ governs the frequency of the oscillatory parts. The solutions given in Tables 1-3 are the most general solutions.
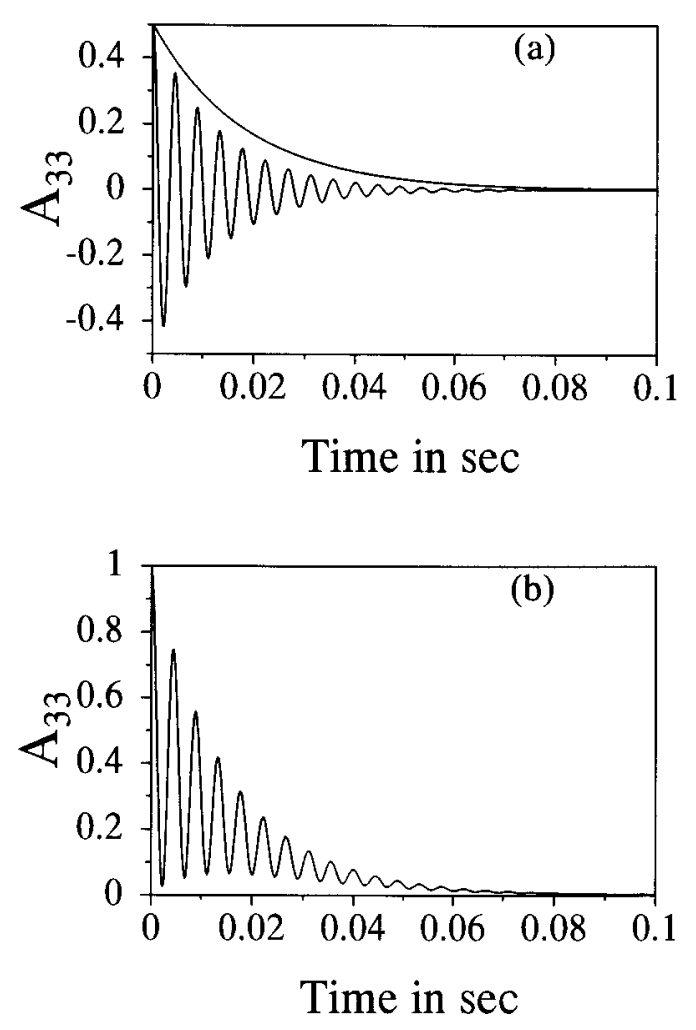

Figure 4 Oscillatory and nonoscillatory parts of $\boldsymbol{A}_{33}$ (Table 1). (a) Separately and (b) added together, for the same values of constants as in Fig. 2. For these values, the parameters $Z_{1}$ and $Z_{2}$ are obtained as $Z_{1}=0.5024$ and $Z_{2}=$ 0.0398. The sine part is small and $\boldsymbol{A}_{33}$ behaves similarly to $\boldsymbol{A}_{11}$ (Fig. 2). 

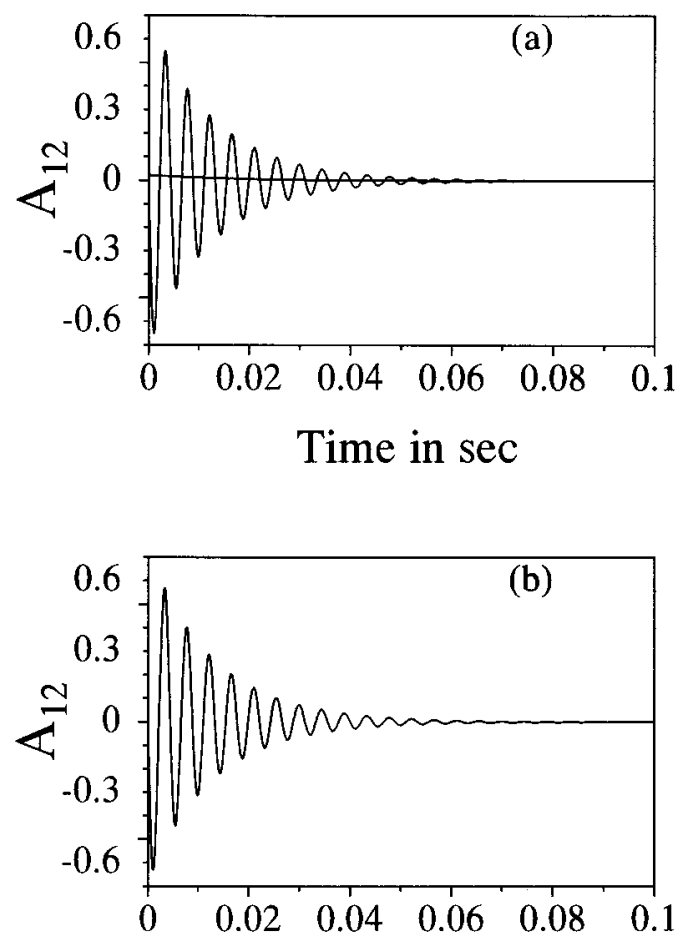

Time in sec

Figure 5 Oscillatory and nonoscillatory parts of $\boldsymbol{A}_{12}$ (Table 1). (a) Separately and (b) added together, for the same values of constants as in Fig. 2. For these values the parameters $N_{1}$ and $N_{2}$ are obtained as $N_{1}=-0.0225$ and $N_{2}$ $=0.7092 . A_{12}$ has a large oscillatory sine part only.

Equation [9] expresses the solution of the generalized Bloch equations in a compact, convenient form and clearly separates the time development of the initial state and that of the steady state.

\section{DISCUSSION}

From the form of the various matrix elements, it is seen that $\mathbf{A}$ is a magnitude symmetric matrix, $\left|\boldsymbol{A}_{i j}\right|$ $=\left|\boldsymbol{A}_{j i}\right|$, such that the amounts of $u_{0}$ and $m_{0}$, which contribute to $v$ are equal and opposite to the contribution of $v_{0}$ to $u$ and $\boldsymbol{M}_{z}\left(\boldsymbol{A}_{12}=-\boldsymbol{A}_{21}\right.$ and $\left.\boldsymbol{A}_{23}=\boldsymbol{A}_{32}\right)$. Also, the amount of $m_{0}$ that contributes to $u$ is equal to the contribution of $u_{0}$ to $\boldsymbol{M}_{z}\left(\boldsymbol{A}_{13}=\boldsymbol{A}_{31}\right)$.

Each diagonal element of $\mathbf{A}$ is in general a biexponential oscillatory decay from one to zero. Each off-diagonal element of $\mathbf{A}$ is in general a biexponential oscillatory term, which starts from zero, grows to a maximum, and decays to zero. The off-diagonal elements represent the coupling and interconversion of each magnetization component and are the most crucial parts of the solution. In the absence of these off-diagonal elements, each initial magnetization component will decay independent of others. Each element of the diagonal matrix $\mathbf{B}$ represents a biexponential oscillatory growth from zero to one. Because $\mathbf{B}$ is diagonal, each steady-state component grows independently of others. This is a consequence of the fact that the inhomogeneous term is contained only in one of the Bloch equations, that is, in the $\boldsymbol{M}_{z}$ equation. If the inhomogeneities were present in other equations as well, $\mathbf{B}$ would be nondiagonal and the steady state also would evolve in a coupled manner. These features of the various matrix elements lead to the following interpretation of the solutions of the Bloch equations.

Because $\mathbf{A}$ and $\mathbf{B}$ are not coupled, the initial state and the steady state evolve independently. The various initial magnetization components oscillate, interconvert among one another and decay biexponentially to zero; this is represented by the matrix A. The steady-state values also grow independently of the initial state and are independent of one another
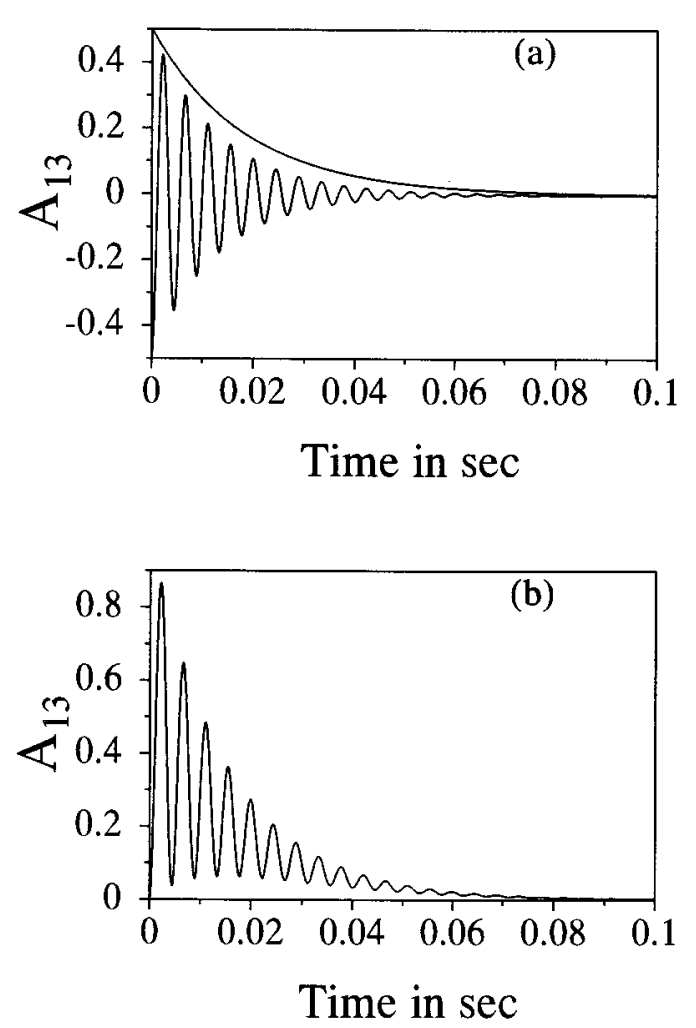

Figure 6 Oscillatory and nonoscillatory parts of $\boldsymbol{A}_{13}$ (Table 1). (a) Separately and (b) added together, for the same values of constants as in Fig. 2. For these values, the parameters $N_{3}$ and $N_{4}$ are obtained as $N_{3}=0.5014$ and $N_{4}$ $=-0.008 . A_{13}$ has large nonoscillatory and oscillatory cosine parts. The oscillatory sine part is small. 

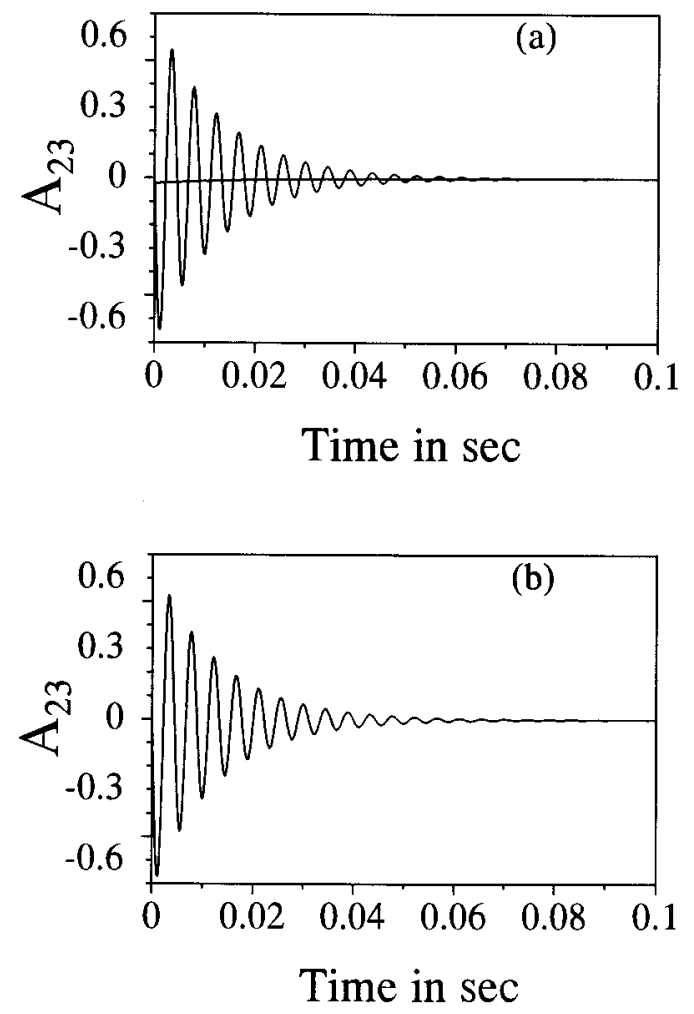

Figure 7 Oscillatory and nonoscillatory parts of $\boldsymbol{A}_{23}$ (Table 1). (a) Separately and (b) added together, for the same values of constants as in Fig. 2. For these values, the parameters $N_{5}$ and $N_{6}$ are obtained as $N_{5}=0.0226$ and $N_{6}$ $=0.7085 . A_{23}$ thus has a large oscillatory sine part only, similar to $\boldsymbol{A}_{12}$ (Fig. 5).

(B is diagonal); they grow from zero to their final values.

The above separation of the solutions of the Bloch equation into two terms not only provides a clear insight about the time development of various magnetization components, but it is also achievable in 2D experiments. This is best illustrated by the simplest experiment, involving inversion-recovery of $z$ magnetization in the absence of an rf field (Fig. 1). One should interpret this experiment as one in which the inverted magnetization decays to zero and a new magnetization grows from zero to one. In a onedimensional (1D) transient nuclear Overhauser effect (NOE) experiment, both components are inseparable and contribute to the total NOE. In a $2 \mathrm{D}$ NOE spectroscopy (NOESY) experiment only the former (A part) contributes to the diagonal and cross-peaks; the latter (B part) contributes to the axial peaks. Therefore, the magnitude of the observed NOE in a 1D transient NOE experiment is twice that in 2D NOESY (B. D. Nageswara Rao, personal communication).

\section{RESULTS}

For illustration, the time development of the various elements of $\mathbf{A}$ and $\mathbf{B}$ are shown for a specific value of the off-resonance $\mathrm{rf}$ field and representative values of $T_{1}$ and $T_{2}$. The values chosen in Figs. 2-12 are $\omega_{1}=$ $1 \mathrm{KHz}, \delta=1 \mathrm{KHz}, T_{1}=0.1 \mathrm{~s}$, and $T_{2}=0.01 \mathrm{~s}$. For these parameters one obtains the following eigenvalues of the solution: $\lambda_{1}=-55.04 \mathrm{~s}^{-1} ; \mu_{2}=-32.48$ $\mathrm{s}^{-1}$; and $\mu_{3}=-1413.30 \mathrm{~s}^{-1}$.

In Fig. 2(a), the oscillatory and nonoscillatory parts of $\boldsymbol{A}_{11}$ are shown separately, and the total value of $\boldsymbol{A}_{11}$ is shown in Fig. 2(b). Because, for the above parameters, $U_{2}$ is very small, and $U_{1}=0.5$, there is a significant nonoscillatory part and an oscillatory part that is mainly a cosine term.

In Fig. 3, $\boldsymbol{A}_{22}$ is shown, and, because $V_{1}$ and $V_{2}$ are both very small, the nonoscillatory part and the sine part are small; $\boldsymbol{A}_{22}$ contains mainly the decaying cosine part. $\boldsymbol{A}_{33}$ as shown in Fig. 4 has a behavior similar to $\boldsymbol{A}_{11}$; because $Z_{2}$ is very small.

The off-diagonal term $\boldsymbol{A}_{12}$ shows (Fig. 5) mainly
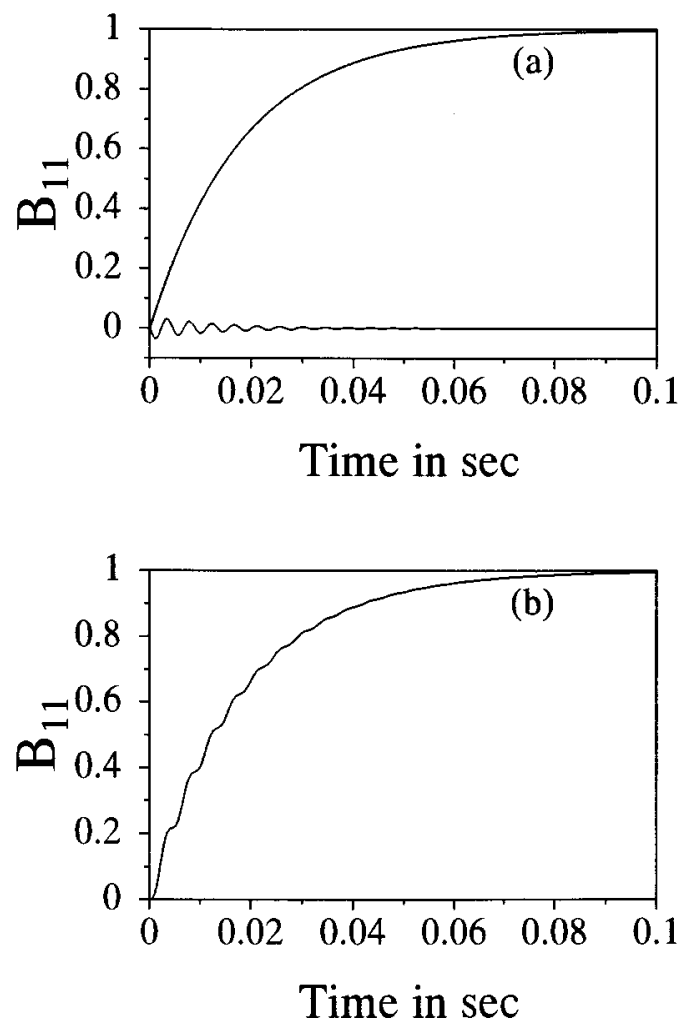

Figure 8 Oscillatory and nonoscillatory parts of $\boldsymbol{B}_{11}$ (Table 2). (a) Separately and (b) added together, for the same values of constants as in Fig. 2. For these values, the parameters $\boldsymbol{M}_{1}$ and $\boldsymbol{M}_{2}$ are obtained as $\boldsymbol{M}_{1}=1.0021$ and $\boldsymbol{M}_{2}=0.039$. Thus $\boldsymbol{B}_{11}$ has a large nonoscillatory part and a small oscillatory sine part. 


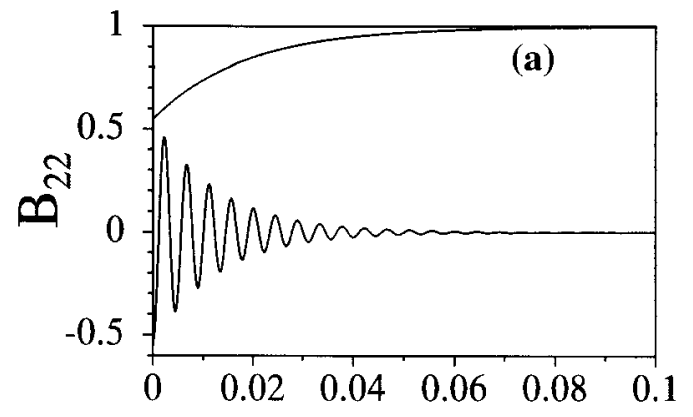

Time in sec

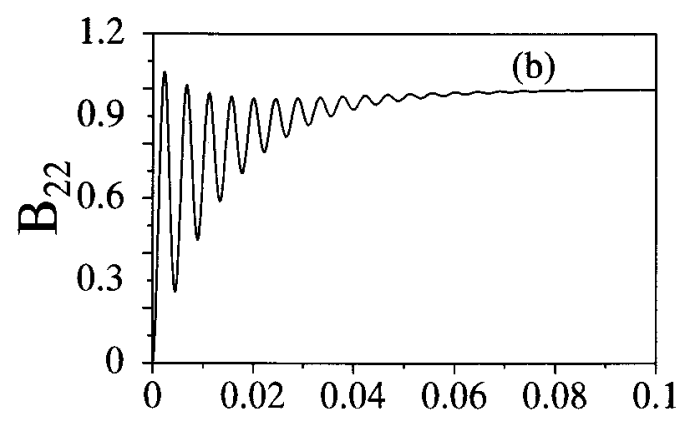

Time in sec

Figure 9 Oscillatory and nonoscillatory parts of $\boldsymbol{B}_{22}$ (Table 2). (a) Separately and (b) added together, for the same values of constants as in Fig. 2. For these values, the parameters $\boldsymbol{M}_{3}$ and $\boldsymbol{M}_{4}$ are obtained as $\boldsymbol{M}_{3}=4.5139$ and $\boldsymbol{M}_{4}=0.0491$. Thus $\boldsymbol{B}_{22}$ has a large nonoscillatory part, a large oscillatory cosine part, and a small oscillatory sine part.

the oscillatory sine part because $N_{1}$ is very small: The term $\boldsymbol{A}_{13}$ (Fig. 6) has a very small sine part $\left(\boldsymbol{N}_{4}\right.$ is small). The term $\boldsymbol{A}_{23}$ (Fig. 7) has a behavior similar to $\boldsymbol{A}_{13}$. Because for the above parameters $\boldsymbol{M}_{1} \approx 1$ and $\boldsymbol{M}_{2}$ is small, the term $\boldsymbol{B}_{11}$ has a large nonoscillatory growth and a small decaying oscillatory part that is mainly a sine term (Fig. 8). Because $\boldsymbol{M}_{3}$ is large and $\boldsymbol{M}_{4}$ is small, $\boldsymbol{B}_{22}$ has a large nonoscillatory part that grows from (1- $\boldsymbol{M}_{3}$ ) to unity, compensated for by a large oscillatory cosine part, which decays from $\left(\boldsymbol{M}_{3}\right.$ - 1) to zero, and a small oscillatory sine part, which decays from $\boldsymbol{M}_{4}$ to zero (Fig. 9). The value of $\boldsymbol{B}_{22}$ oscillates beyond unity even though its value at $t=\infty$ is unity. At first, this could look nonphysical, but the value of $\boldsymbol{B}_{22}$ is multiplied by $v^{\infty}$ such that $\boldsymbol{B}_{22} \cdot v^{\infty}$ never goes beyond unity. $\boldsymbol{B}_{33}$ has a behavior similar to $\boldsymbol{B}_{11}$ (Fig. 10). Although $\boldsymbol{B}_{11}$ and $\boldsymbol{B}_{33}$ show mainly a monotonic growth from 0 to $1, \boldsymbol{B}_{22}$ has a nonmonotonic growth from zero to unity for the chosen parameters.
The phase trajectories of the decay of initial magnetization components for a general initial state given by $u_{0}=v_{0}=m_{0}=0.1$ are shown in Fig. 11; which show that $u_{0}, v_{0}$, and $m_{0}$ decay in a coupled manner. The decay of $u_{0}, v_{0}$, and $m_{0}$ and the growth of $u^{\infty}, v^{\infty}$, and $M^{\infty}$ are given, respectively, by Eqs. [11]-[16]:

$$
\begin{gathered}
u_{0}(t)=\boldsymbol{A}_{11}(t) u_{0}+\boldsymbol{A}_{12}(t) v_{0}+\boldsymbol{A}_{13}(t) m_{0} \\
v_{0}(t)=\boldsymbol{A}_{21}(t) u_{0}+\boldsymbol{A}_{22}(t) v_{0}+\boldsymbol{A}_{23}(t) m_{0} \\
m_{0}(t)=\boldsymbol{A}_{31}(t) u_{0}+\boldsymbol{A}_{32}(t) v_{0}+\boldsymbol{A}_{33}(t) m_{0} \\
u^{\infty}(t)=\boldsymbol{B}_{11}(t) u^{\infty} \\
v^{\infty}(t)=\boldsymbol{B}_{22}(t) v^{\infty} \\
M_{z}^{\infty}(t)=\boldsymbol{B}_{33}(t) M_{z}^{\infty}
\end{gathered}
$$

They are shown in Fig. 12. Figure 12 shows that $u_{0}(t), v_{0}(t)$, and $m_{0}(t)$ decay in an oscillatory manner from their respective initial values to zero and that

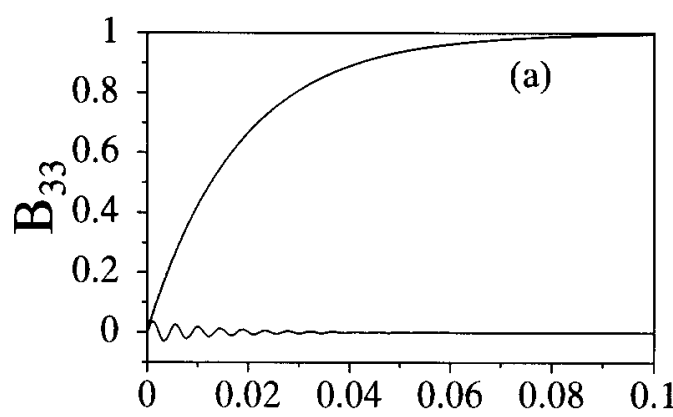

Time in sec

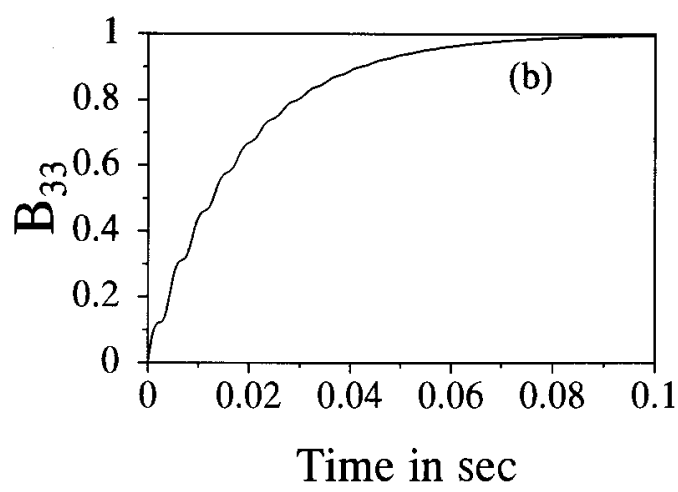

Figure 10 Oscillatory and nonoscillatory parts of $\boldsymbol{B}_{33}$ (Table 2). (a) Separately and (b) added together, for the same values of constants as in Fig. 2. For these values, the parameters $\boldsymbol{M}_{5}$ and $\boldsymbol{M}_{6}$ are obtained as $\boldsymbol{M}_{5}=1.004$ and $\boldsymbol{M}_{6}$ $=-0.039$. $\boldsymbol{B}_{33}$ has a behavior similar to $\boldsymbol{B}_{11}$ (Fig. 9). 
$u^{\infty}(t), v_{0}^{\infty}(t)$, and $M_{z}^{\infty}(t)$ grow from zero to their respective final values also in an oscillatory manner.

The behavior of the various elements of $\mathbf{A}$ and $\mathbf{B}$ for various limiting cases such as on-resonance $\mathrm{rf}$ field, weak rf field, or neglect of $T_{1}$ or $T_{2}$ are extremely useful and illustrative. These have been discussed in detail in Ref. 12 and are discussed briefly here.

\section{Case 1: On-Resonance rf Field}

The solution of Bloch equations for the on-resonance case $(\delta=0)$ becomes simple, as $u$ is not coupled to $v$ and $\boldsymbol{M}_{\mathrm{z}}$. Therefore, $u_{0}$ decays with a single exponential given by $\exp \left(-t / T_{2}\right)$ (Table 5-7). This follows from the facts that in this limit, $\boldsymbol{A}_{12}=\boldsymbol{A}_{21}=\boldsymbol{A}_{13}=$ $\boldsymbol{A}_{31}=0 ; \boldsymbol{A}_{11}$ is reduced to $\exp \left(-t / T_{2}\right)$; and, because in this limit $u^{\infty}=0, \boldsymbol{B}_{11}$ is not relevant. The solution of $\boldsymbol{v}$ and $\boldsymbol{M}_{z}$ remains coupled, yielding simple roots of the cubic equation (Table 3, Eq. [3-1]) as

$$
\begin{gathered}
\lambda_{1}=-\beta \\
\lambda_{2,3}=\frac{1}{2}[-(\alpha+\beta) \pm i \sqrt{\Delta}]
\end{gathered}
$$

with

$$
\Delta=4 \omega_{1}^{2}-(\alpha-\beta)^{2}
$$

The behavior of $v$ and $\boldsymbol{M}_{z}$ further depends on the strength of the rf field $\left(\omega_{1}\right)$ with respect to the difference of $1 / T_{1}$ and $1 / T_{2}$. Three cases can be distinguished.

(a) Radio Frequency Field Strong Compared to Relaxation, such that $2 \omega_{1}>\left|1 / T_{1}-1 / T_{2}\right|$. In this case $\Delta$ is positive, yielding oscillatory solutions of $v$ and $M_{z}$. $\boldsymbol{A}_{22}, \boldsymbol{A}_{33}$, and $\boldsymbol{A}_{23}$ (Table 5) remain oscillatory but become single-exponentially-decaying functions. $\boldsymbol{B}_{22}$ and $\boldsymbol{B}_{33}$ also remain oscillatory but become singleexponentially-growing functions from zero to 1 .

(b) Radio Frequency Field Comparable to Relaxation, such that $2 \omega_{1}=\left|1 / T_{1}-1 / T_{2}\right|$. In this case $\Delta$

Figure 11 Phase trajectories of the decay of the initial magnetization components for $u_{0}=v_{0}=m_{0}=0.1 ; \delta=$ $1 \mathrm{KHz} ; \omega_{1}=1 \mathrm{KHz} ; T_{1}=0.1 \mathrm{~s}$; and $T_{2}=0.01 \mathrm{~s}$. (a) $u_{0}(t)$ $-v_{0}(t)$; (b) $u_{0}(t)-m_{0}(t)$; and (c) $v_{0}(t)-m_{0}(t)$, where $u_{0}(t)$, $v_{0}(t)$, and $m_{0}(t)$ are given by Eqs. [11]-[13]. All the initial values decay to zero. The filled circles represent the initial state; crosses represent the final state. The $\mathbf{B}$ part is not included in these diagrams.
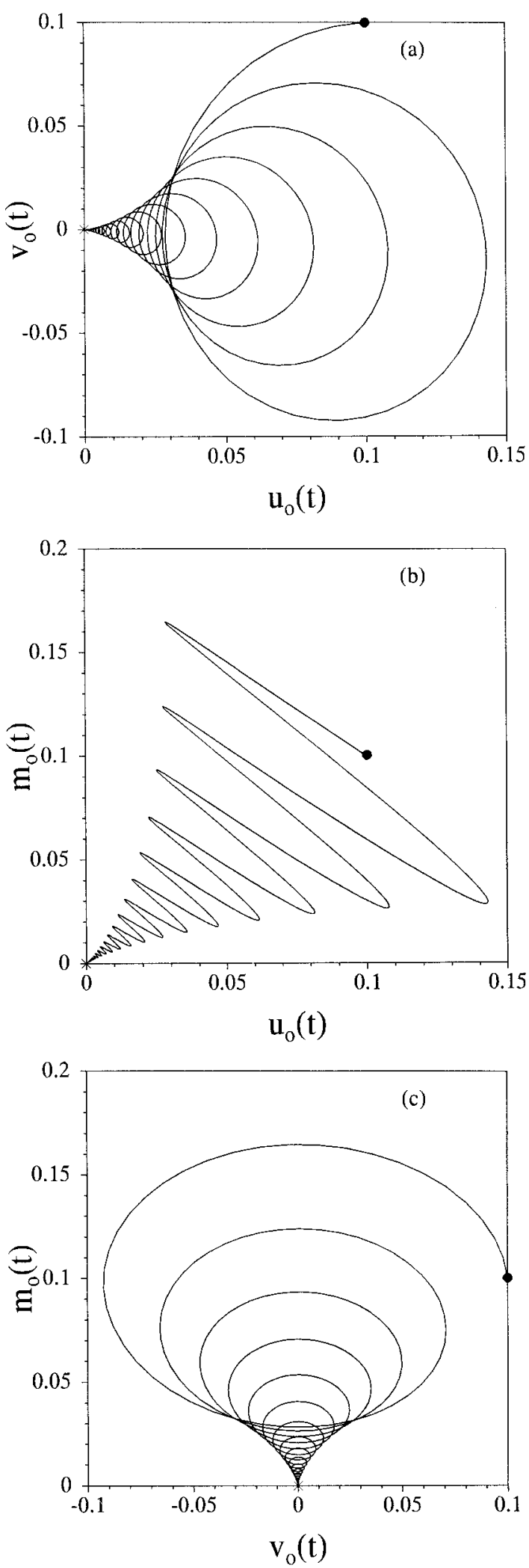

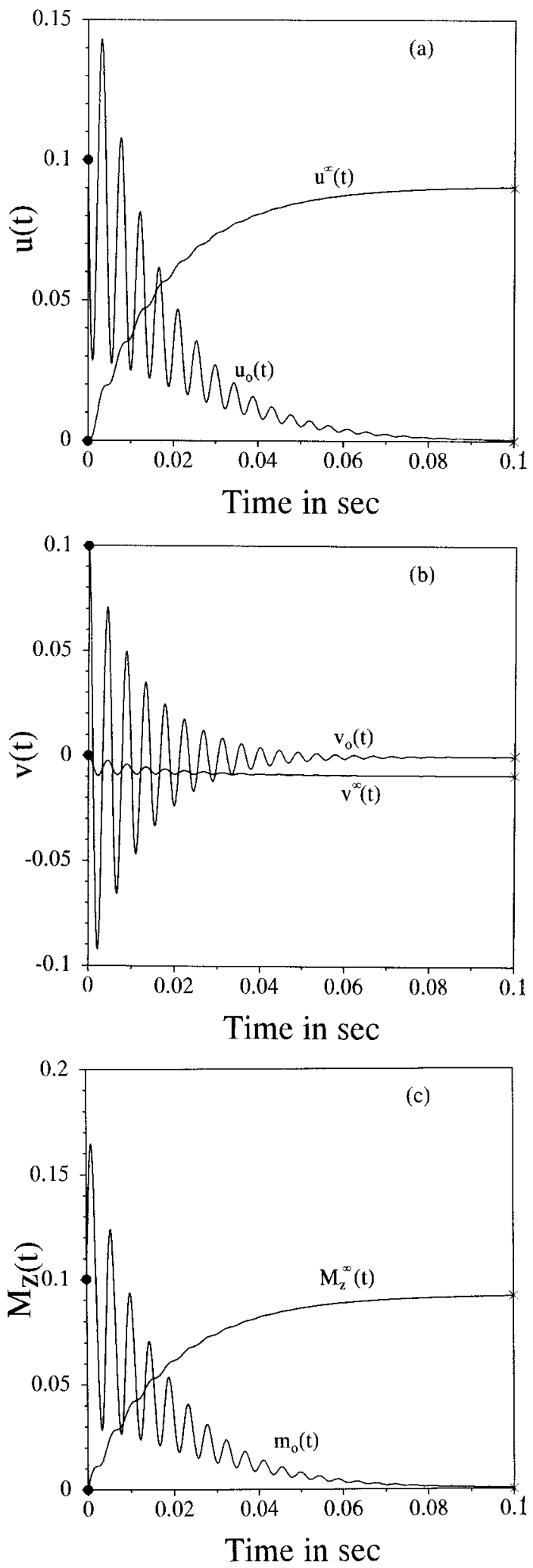

$=0$. This means that the $\mathrm{rf}$ field is equal to linewidth for $T_{2} \ll T_{1}$. This yields simple exponential evolution of $\boldsymbol{v}^{\infty}$ and $\boldsymbol{M}_{z}^{\infty}$ as well as a simple exponential uncoupled evolution of $v_{0}$ and $m_{0}$ with a rate constant that is an average of $1 / T_{1}$ and $1 / T_{2}$ (Table 6). For $T_{1}=$ $T_{2}$, this solution is also the on-resonance solution of Bloch equations in the absence of rf fields.

(c) Radio Frequency Field Weak Compared to Relaxation, such that $2 \omega_{1}<\left|1 / T_{1}-1 / T_{2}\right|$. In this case $\Delta$ is negative, making all three roots of Eq. [11] real. The various elements of $\mathbf{A}$ and $\mathbf{B}$ matrices (Table 7) do not oscillate and show biexponential behavior. However, $\boldsymbol{v}$ and $\boldsymbol{M}_{z}$ are coupled $\left(\boldsymbol{A}_{23} \neq 0\right)$ and interconvert.

In the presence of $\mathrm{rf}$, the coupled evolution of transverse and longitudinal magnetization in Bloch equations also leads to a coupled relaxation of these components. Although the general result is contained in Tables 1 and 2, the specific case of $\delta=0$ (Tables 5-7) demonstrates this explicitly. In the three cases (a), (b) and (c) (Tables 5-7), the $\boldsymbol{v}$ and $\boldsymbol{M}_{z}$ components have exponential behavior governed by $(\alpha+\beta) / 2,(\alpha$ $-\beta) / 2$ and $2 \omega_{1} / \sqrt{\bar{\Delta}}$. For decay of $v_{0}$ and $m_{0}$ in (a) and (c) when the rf field is strong or weak compared to relaxation (Tables 5 and 7), this is not surprising; because their evolution is also coupled $\left(\boldsymbol{A}_{23} \neq 0\right)$. On the other hand, it is interesting that, when the rf field is comparable to relaxation (Table 6), the rates continue to be coupled, given by $(\alpha+\beta) / 2$, even when $\boldsymbol{A}_{23}=0$. Similarly, because the growth of the steady state is not coupled (B is diagonal), the rate of growth of $\boldsymbol{B}_{22}$ and $\boldsymbol{B}_{33}$ being coupled is interesting. Although these magnetization components grow in an uncoupled manner, their growth rates are a mixture of $T_{1}$ and $T_{2}$. The rates are equal mixtures for the first two cases, (a) and (b) but in the third case, (c) the mixture is governed by the factors $(\alpha+\beta) / \sqrt{\Delta^{\prime}}$ and $\left(\beta^{2}-\alpha \beta\right.$ $\left.-2 \omega_{1}^{2}\right) / \beta \sqrt{\Delta^{\prime}}$ (Table 7). In the limit $\omega_{1} \rightarrow 0, \boldsymbol{B}_{22}$ is not relevant because $v^{\infty}=0$, and the growth rate of $\boldsymbol{M}_{z}^{\infty}$ becomes $1 / T_{1}$, as it should. The growth rates of $u^{\infty}(t)$ and $\boldsymbol{M}^{\infty}(t)$ in Fig. 12, which has $\delta \neq 0$, are more complex (given by Tables 2 and 3). However, it can be clearly seen that the rates are between $1 / T_{1}$ and $1 / T_{2}$.

Figure 12 Time evolution of the initial and final state of each of the magnetization components for the parameters of Fig. 11. Time evolution of (a) $u_{0}(t)$ and $u^{\infty}(t)$; (b) $v_{0}(t)$ and $v^{\infty}(t)$; and (c) $m_{0}(t)$ and $\boldsymbol{M}_{z}^{\infty}(t)$. The filled circles represent the initial state and crosses the state at $t=0.1 \mathrm{~s}$. For the above parameters, the steady-state values are $u^{\infty}=$ $-0.0908, \mathrm{v}^{\infty}=-0.0091$, and $\boldsymbol{M}_{z}^{\infty}=0.0917$. 


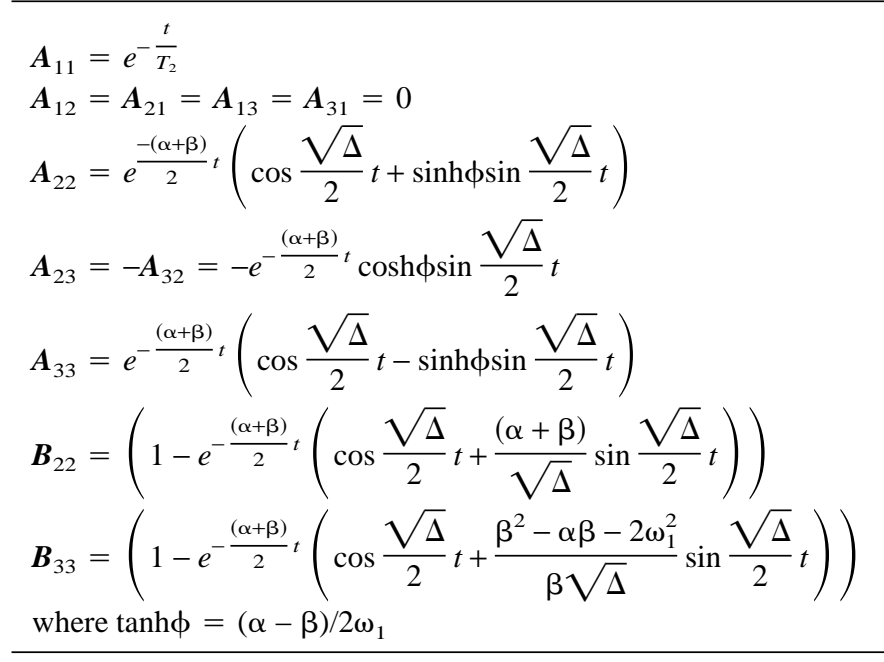

\section{Case 2: Off-Resonance, Weak rf Field}

For small values of rf fields, retaining terms proportional to $\omega_{1}$, and neglecting terms of the order of $\omega_{1}^{2}$ in Table 3, Eq. [3-2], one obtains simpler definitions for the constants $\mathrm{a}, \mathrm{b}$, and $\mathrm{c}$ of the cubic equation (Table 3, Eq. [3-1]), which on substitution yield a straightforward factorization of Eq. [3-1] to give

$$
\begin{gathered}
\lambda_{1}=-\alpha \\
\mu_{2}=-\beta \\
\mu_{3}=\delta
\end{gathered}
$$

The various elements of $\mathbf{A}$ and $\mathbf{B}$ are listed in Table 8 . The expressions of $\boldsymbol{M}_{1}, \boldsymbol{M}_{2}, \boldsymbol{M}_{3}$, and $\boldsymbol{M}_{4}$ can be obtained from Table 3 by making use of Eq. [13].

Here, all the elements of the $\mathbf{A}$ matrix are nonzero, and hence $u_{0}, v_{0}$, and $m_{0}$ decay in a coupled manner. The elements oscillate at the frequency of offresonance value. All the diagonal elements of $\mathbf{A}$, the off-diagonal element $\boldsymbol{A}_{12}$, and $\boldsymbol{B}_{33}$ have singleexponential behavior; the off-diagonal elements $\boldsymbol{A}_{13}$ and $\boldsymbol{A}_{23}$ of $\mathbf{A}, \boldsymbol{B}_{11}$, and $\boldsymbol{B}_{22}$ have biexponential behavior. This solution is more general than the solution for weak rf fields given by Slichter (8).

Table 6 Elements of $A$ and $B$ for $\delta=0$ and $\Delta=0$

$$
\begin{aligned}
& A_{11}=e^{-\frac{t}{T_{2}}} \\
& \boldsymbol{A}_{12}=\boldsymbol{A}_{21}=\boldsymbol{A}_{13}=\boldsymbol{A}_{31}=\boldsymbol{A}_{23}=\boldsymbol{A}_{32}=0 \\
& \boldsymbol{A}_{22}=e^{\frac{-(\alpha+\beta)}{2} t} \\
& \boldsymbol{A}_{33}=e^{\frac{-(\alpha+\beta)}{2} t} \\
& \boldsymbol{B}_{22}=B_{33}=1-e^{\frac{-(\alpha+\beta)}{2} t}
\end{aligned}
$$

In addition to assuming a weak rf field, Slichter assumes that $\boldsymbol{M}_{\mathrm{z}}(\mathrm{t})=\boldsymbol{M}_{0}(8)$. This has the following consequences; first, it assumes that at $t=0$ the $z$ magnetization is nearly at equilibrium and the $x$ and $y$ components have small values, $u_{0}$ and $v_{0}$. Table 8 , on the other hand, describes evolution of any arbitrary initial condition. The second consequence is that the evolution of $\boldsymbol{M}_{z}$ is decoupled from that of $u$ and $v$. This condition $\boldsymbol{M}_{z}(t)=\boldsymbol{M}_{0}$ is not a parametric condition and cannot be substituted in the general solution of Table 8. One must ab initio solve the Bloch equations with the condition, $\boldsymbol{M}_{z}(t)=\boldsymbol{M}_{0}$, decoupling the equations, as has been done by Slichter $(8,12)$.

For $\omega_{1}=0$, the solution of Table 8 reduces to an off-resonance rotating-frame solution of Bloch equations, such that $u_{0}$ and $v_{0}$ decay in a coupled manner $\left(\boldsymbol{A}_{12} \neq 0\right)$ and $m_{0}$ decays independently $\left(\boldsymbol{A}_{13}=\boldsymbol{A}_{23}\right.$ $=0)$. In this limit, $u^{\infty}=v^{\infty}=0$ and hence $\boldsymbol{B}_{11}$ and $\boldsymbol{B}_{22}$ are not relevant. $M_{z}^{\infty}(t)$ shows single-exponential growth, at the same rate as the decay of $m_{0}$, described by the curves $b, e$ and $a, d$, respectively, of Fig. 1 .

\section{Case 3: Neglecting Relaxation}

For this case, $1 / T_{1}=1 / T_{2}=0$. In this limit, one obtains nondecaying oscillatory behavior for all the elements of the A matrix. All the elements of the $\mathbf{B}$ matrix are zero (Table 9). If, in addition, one assumes on-resonance $(\delta=0)$, then $v_{0}$ and $m_{0}$ show coupled oscillations since $\boldsymbol{A}_{23} \neq 0$ with $u_{0}$ remaining unchanged $\left(\boldsymbol{A}_{11}=1, \boldsymbol{A}_{12}=\boldsymbol{A}_{13}=0\right)$ and spin locked along the rf field. However, if $\omega_{1}=0$, then $u_{0}$ and $v_{0}$ show coupled oscillations $\left(\boldsymbol{A}_{12} \neq 0\right)$, and $m_{0}$ remains unchanged because $\boldsymbol{A}_{33}=1$ and $\boldsymbol{A}_{13}=\boldsymbol{A}_{23}=0$. 


$$
\begin{aligned}
& \boldsymbol{A}_{11}=e^{-\frac{t}{T_{2}}} \\
& \boldsymbol{A}_{12}=\boldsymbol{A}_{21}=\boldsymbol{A}_{13}=\boldsymbol{A}_{31}=0 \\
& \boldsymbol{A}_{22}=e^{\frac{-(\alpha+\beta)}{2} t}\left(\cosh \frac{\sqrt{\Delta^{\prime}}}{2} t+\cosh \phi^{\prime} \sinh \frac{\sqrt{\Delta^{\prime}}}{2} t\right) \\
& \boldsymbol{A}_{23}=-\boldsymbol{A}_{32}=-e^{-\frac{(\alpha+\beta)}{2} t} \sinh \phi^{\prime} \sinh \frac{\sqrt{\Delta^{\prime}}}{2} t \\
& \boldsymbol{A}_{33}=e^{-\frac{(\alpha+\beta)}{2} t} t\left(\cosh \frac{\sqrt{\Delta^{\prime}}}{2} t-\cosh \phi^{\prime} \sinh \frac{\sqrt{\Delta^{\prime}}}{2} t\right) \\
& \boldsymbol{B}_{22}=\left(1-e^{-\frac{(\alpha+\beta)}{2} t}\left(\cosh \frac{\sqrt{\Delta^{\prime}}}{2} t+\frac{(\alpha+\beta)}{\sqrt{\Delta^{\prime}}} \sinh \frac{\sqrt{\Delta^{\prime}}}{2} t\right)\right) \\
& \boldsymbol{B}_{33}=\left(1-e^{-\frac{(\alpha+\beta)}{2} t}\left(\cosh \frac{\sqrt{\Delta^{\prime}}}{2} t+\frac{\beta^{2}-\alpha \beta-2 \omega_{1}^{2}}{\beta \sqrt{\Delta^{\prime}}} \sinh \frac{\sqrt{\Delta^{\prime}}}{2} t\right)\right) \\
& \text { where tanh } \phi^{\prime}=2 \omega_{1} /(\alpha-\beta) \text { and } \Delta^{\prime}=-\Delta
\end{aligned}
$$

\section{Table 8 Elements of A and B for weak rf Field}

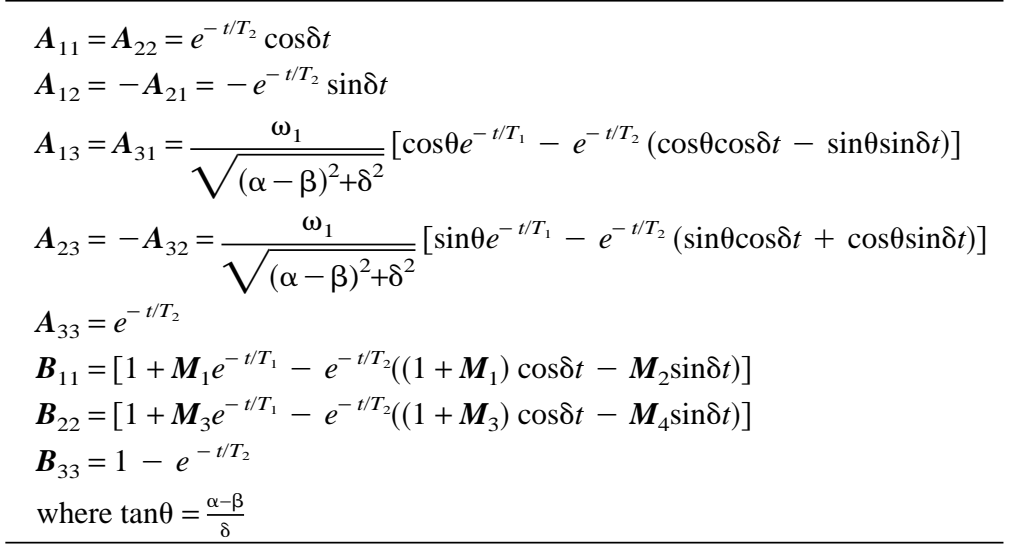

\section{Table 9 Elements of A and B}

\section{Neglecting Relaxation}

$$
\begin{aligned}
\boldsymbol{A}_{11} & =1-2 \sin ^{2}\left(\theta_{e}\right) \sin ^{2}\left(\frac{\omega_{e}}{2} t\right) \\
\boldsymbol{A}_{12} & =-\boldsymbol{A}_{21}=-\sin \left(\theta_{e}\right) \sin \left(\omega_{e} t\right) \\
\boldsymbol{A}_{131} & =\boldsymbol{A}_{31}=\sin \left(2 \theta_{e}\right) \sin ^{2}\left(\frac{\omega_{e}}{2} t\right) \\
\boldsymbol{A}_{22} & =\cos \left(\omega_{e} t\right) \\
\boldsymbol{A}_{23} & =-\boldsymbol{A}_{32}=-\cos \left(\theta_{e}\right) \sin \left(\omega_{e} t\right) \\
\boldsymbol{A}_{33} & =1-2 \cos ^{2}\left(\theta_{e}\right) \sin ^{2}\left(\frac{\omega_{e}}{2} t\right) \\
\boldsymbol{B}_{11} & =\boldsymbol{B}_{22}=\boldsymbol{B}_{33}=0
\end{aligned}
$$

where $\omega_{e}=\sqrt{\delta^{2}+\omega_{1}^{2}}$ and $\tan \theta_{e}=\frac{\delta}{\omega_{1}}$

\section{CONCLUSIONS}

The solutions of the Bloch equations presented here show that, in general, every initial state decays in a biexponential oscillatory manner while the steady state independently grows from zero-also in an oscillatory, biexponential manner. The decay of the initial state takes place independently of the growth of the steady state, and each component of the steady state grows independently of the other components. The decay of the initial state and the growth of the steady state can be separated by 2D experiments, where the former gives the diagonal and cross-peaks and the latter gives the axial peaks. 


\section{ACKNOWLEDGMENT}

Discussions with the NMR group at I.I.Sc., Bangalore, especially K. V. Ramanathan, are gratefully acknowledged.

\section{REFERENCES}

1. F. Bloch, "Nuclear Induction," Phys. Rev., 1946, 70, 460-474.

2. H. C. Torrey, "Transient Nutations in Nuclear Magnetic Resonance," Phys. Rev., 1949, 76, 1059-1068.

3. A. Abragram, Principles of Nuclear Magnetic Resonance, Oxford University Press, London, 1961, pp. $45-46$.

4. J. McConnell, The Theory of Nuclear Magnetic Relaxation in Liquids, Cambridge University, London, 1987, p. 9 .

5. P. W. Atkins, K. A. McLauchlan, and P. W. Percival, "Electron Spin-Lattice Relaxation Times from the Decay of E.S.R. Emission Spectra,' Mol. Phys., 1973, 25, 281-296.

6. J. B. Pedersen, "Theory of Transient Effects in Time Resolved ESR Spectroscopy,' J. Chem. Phys., 1973 59, 2656-2667.

7. J. D. Roberts, "The Bloch Equations. How to Have Fun Calculating What Happens in NMR Experiments with a Personal Computer,' Concepts Magn. Reson., 1991, 3, 27-45.

8. C. P. Slichter, Principles of Magnetic Resonance, 3rd ed., Springer Verlag, New York, 1989, pp. 35-36.

9. R. V. Mulkern and M. L. Williams, "The General Solution to the Bloch Equation with Constant rf and Relaxation Terms: Application to Saturation and Slice Selection," Med. Phys., 1993, 20, 5-13.

10. G. A. Morris and P. B. Chilvers, "General Analytical Solutions of the Bloch Equations," J. Magn. Reson., 1994, A 107, 236-238.

11. P. J. Hore and K. A. McLauchlan, "Chemically Induced Dynamic Electron Polarization (CIDEP) and Spin-Relaxation Measurements by Flash-Photolysis
Electron Paramagnetic Resonance Methods,' J. Magn. Reson., 1979, 36, 129-134.

12. P. K. Madhu and Anil Kumar, "Direct Cartesian Space Solutions of the Generalized Bloch Equations in the Rotating Frame," J. Magn. Reson., 1995, A 114, $201-$ 212.

13. S. Barnard and J. M. Child, Higher Algebra, Macmillan, London, 1960.

14. W. H. Beyer, Ed., Standard Mathematical Tables, 25th ed., CRC, Boca Raton, FL, 1978.

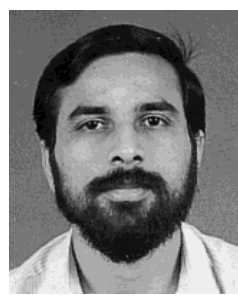

P. K. Madhu did his undergraduate studies at the Indian Institute of Technology, Madras, with specialization in condensed-matter physics. Currently he is doing graduate studies at the Indian Institute of Science, Bangalore, with Prof. Anil Kumar in the broad area of nuclear magnetic resonance. His research interests are: effects of cross-correlation on relaxation, NMR studies of biomolecules, and solid-state NMR.

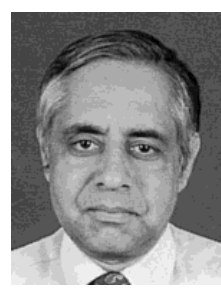

Anil Kumar is professor and currently chairman of the Department of Physics and also a professor at the Sophisticated Instruments $\mathrm{Fa}$ cility (a national NMR facility) at the Indian Institute of Science in Bangalore. He holds a Ph.D. from the Indian Institute of Technology, Kanpur (1969, supervised by Prof B.D.N. Rao). He did postdoctoral work in the U.S.A. at the Georgia Institute of Technology in Atlanta (1969-1970) with Prof. Sidney Gordon; at the University of North Carolina at Chapel Hill (1970-1972) with Prof. C. S. Johnson, Jr.; and in Switzerland at E.T.H. Zürich (1973-1976) with Prof. Richard R. Ernst. Prof. Kumar joined the institute in Bangalore in 1977 and became an assistant professor in 1982. He was associate professor in 1984, and a professor in 1990. He was a visiting professor at the University of North Carolina during 198990 and a research associate at E.T.H. Zürich during 1979-1980 (jointly with Prof. R. R. Ernst and Prof. K. Wüthrich). Prof. Kumar's research is on NMR technique development and relaxation studies. He has worked on double-resonance techniques, twodimensional NMR and its applications to biomolecules, and Fourier NMR imaging. Prof. Kumar has published several papers dealing with strong coupling effects in NMR. 